\title{
Personal Learning Environments in a Global Higher Engineering Education Web 2.0 Realm
}

\author{
Denis Gillet \\ School of Engineering \\ École Polytechnique Fédérale de Lausanne (EPFL) \\ Lausanne, Switzerland \\ denis.gillet@epfl.ch
}

\author{
Effie L-C. Law and Arunangsu Chatterjee \\ Department of Computer Science \\ University of Leicester \\ LE1 7RH Leicester, UK \\ elaw@mcs.le.ac.uk and A.Chatterjee@mcs.le.ac.uk
}

\begin{abstract}
This paper presents investigations on formal and informal requirements for personal learning environments taking into account students' personal and social learning practices. The potential of global Web 2.0 educational service bundles and informal learning communities, as well as their recommendation by educators are addressed. A scenario showing how these new paradigms can be integrated in engineering education as a way to bring together personal and social learning practices is drawn.
\end{abstract}

Web 2.0; Personal Learning Environments; Mash up; Social Learning; Personal Learning; Educational Widgets; Learning Communities

\section{INTRODUCTION}

A hidden revolution in higher engineering education is currently underway for multiple reasons. First, the students entering the university are digital natives often with higher technical skills than their educators, who are digital immigrants. Second, Web 2.0 technologies enable students to mash up the learning resources, the learning services and the learning communities of their choice. The same technologies also enable educators to move from blended learning approaches to blended contents (blogs, wiki, repositories) and blended learning environments called personal learning environments [1]. Such environments will progressively replace, or at least complement, learning management systems (LMS) in the coming years in a move towards personal and social learning environments. Third, students can access open learning repositories outside their institutions.

The opportunity for students to build their own learning environments or socio-academic contexts, as well as their own learning networks or communities has always existed at a local level (campus or hometown). People enrolled in the same study programs or courses are used to meeting in social settings to do homework or prepare exams. With the above-mentioned trends, higher engineering education institutions should have a closer look at these informal personal and social spaces and practices, which are expending at a global scale and are giving students access to an unlimited realm of potentially valuable resources and experts. In addition, educational scientists consider the construction of learning environments and the integration of learning communities as being an integral part of the learning process. Especially, it helps students to develop the high-level skills and competences required by their future employers. Hence, a challenge for academic institutions is to integrate in a proper way the students' practices and environments in the existing institutional ones in order to take advantage of them. Another challenge is to support the students in their informal learning practices and in the construction of their learning environments and networks as a next step in increasing digital literacy. Students nowadays need recommendation regarding trusty resources and networks that go beyond the traditional brick-and-mortar universities.

The ROLE European integrated research project (http://www.role-project.eu/) has been investigating since February 2009 the interplay between personal learning and personal learning environments. 16 partners with educational, technological and commercial background collaborate in a multidisciplinary and intercultural manner to sketch the next generation of personal learning environments that can contribute to changes in the education paradigm as described in Sections II and III. The rest of the paper concentrates on results regarding formal and informal requirements for personal learning environments (Sections IV, V and VI), taking into account the peculiarities of personal and social learning. The potential of Web 2.0 service bundles and their recommendation by educators are tackled [2]. A prototype of novel social software fulfilling part of the elicited requirements is described in Section VII. A scenario for integrating these new paradigms in engineering education is drawn in Section VIII before concluding in Section IX.

\section{USER-CENTERED PERSPECTIVE}

The current practice in higher education for faculty members struggling to increase their h-index [3] (or any other fashionable academic metrics) is to concentrate on lectures and a few office hours. Lecturing is the most effective approach from an administrative (well recognized duty) and teaching (best return on effort ratio) point of view. However, its impact on students' learning (knowledge and competences acquisition and consolidation) is rather limited. As a consequence, most of students' learning activities occur through personal work; partially with the help of teaching assistants. In the last 2 decades, technologies were introduced mainly to extend the classroom reach. Nowadays, some lectures are podcasted as example in iTunes $U$ and PowerPoint-like presentations are made available on institutional LMS. In the latter, the students 
can also post their homework assignments without heavy involvement of teachers or institutional staff. Additional interaction occurs between students and educators through email and course-related blogs. Recently, in a move to develop the acquisition of high-level skills and competences, more active learning opportunities like labwork or teamwork have been introduced and are supported by relevant technologies, which are also often integrated in LMS. Due to the lack of adequate institutional resources, the support for such activities - which are highly demanding in terms of coaching - is unfortunately not always sufficient.

To compensate for their increasing isolation [4] and the standardization of the resources, which result from the presence of LMS or just from the lack of sufficient face-to-face interaction with educators, the students are relying more and more on peers and technology of their choice to manage and complete their learning activities in a self-directed manner. As pointed out by Attwell [1], Google is the most used e-learning platform in such a context where students are struggling to find relevant and personalized resources [5] and support.

This trend is counterproductive for both the institutions and the learners. The institutions are undermining their reputation and their attractiveness by losing the control on the resources and their usage. The learners, especially the ones with little social network and limited digital literacy, waste their time in unproductive search rather than focusing on productive activities.

Fortunately and thanks to the spreading of social software platforms and open learning repositories, there is currently an opportunity for institutions and learners to establish a stimulating and constructive interaction to strengthen learning and increase effectiveness. The idea is not for the institutions to invade the social space of the learners, but rather to promote trusty resources and academic presence that can be integrated by the learners themselves in their own spaces and own communities [6].

There are several challenges associated with such a move. First, the social spaces of the students have to be better understood, especially their relations with informal or selfdirected learning [7]. Second, one has to evaluate how engineering education resources can be integrated in such spaces without destroying their unique personal and social nature. In other words, could those personal environments be turned into personal learning environments [8]? Third, all the necessary features to turn personal environments into personal learning ones have to be elicited, taking into account the necessary bridges to link the digital Web 2.0 realm and the physical world that coexist when blending formal and informal education. Finally, the roles the educators and the institutions have to play with respect to this evolution have to be reconsidered carefully. This paper focuses on the third issue, which is discussed in the next sections.

\section{PERSONAL LEARNING ENVIRONMENTS}

Personal Learning Environments (PLE) are not monolithic systems. They can be simply a set of devices, tools, applications, and physical or virtual spaces associated by learners at a specific time, for a specific purpose, and in a given context. A student's desk covered by books and notes, combined with a computer holding a collection of slides and documents with the associated applications for reading and editing, integrating a browser to access the Web or just the institutional LMS, is already a PLE. Such PLE, even though effective, is yet to reap potential benefits of customization and collaboration offered by Web 2.0 technologies. In this paper, we focus especially on the exploration and exploitation of new Web 2.0 features that strengthen the role of the social repositories and communities in self-directed learning, enabling learners to assemble and customize even more effective PLEs. As a consequence, end-user Web 2.0 services, online social spaces and shared applications are especially investigated, with a special focus on platforms where they can be aggregated, integrated, or mashed up.

Approaches and standards for the aggregation and the integration of Web 2.0 components as PLE services bundles have been investigated with an emphasis on interoperability issues [9]. Mashup platforms like iGoogle (www.google.com) or Netvibes (www.netvibes.com) also show a high potential to be exploited as Web 2.0 PLEs. In such framework, Web 2.0 components or services bundled together are often widgets or similar Web artifacts.

As the integration of PLEs relies on tools, artifacts and people collected or invited by users from the whole Webosphere, trust in the quality of the resources, the security of the distributed information storage (especially for personal and competence-related information) and the reputation of the providers or the communities are instrumental in enabling the self-directed repurposing of Web 2.0 spaces and services for learning.

\section{REQUIREMENTS ANALYSIS APPROACHES AND HYPOTHESES}

The design and deployment of Web 2.0 PLEs is a completely new design paradigm for three reasons. First, PLEs are not end products; they are rather contexts or spaces continuously crafted and personalized by individuals according to their goals, interests, activities and even mood. They are not controlled by designers, service providers, or Web masters, but by end users. The role of third parties is somehow limited to providing users with convenient integration and customization solutions. Second, the design and integration processes themselves as well as the appropriation of the PLEs are part of the underlying social or learning activities. In this perspective, design and integration cannot be decoupled from socializing, interaction or learning. Third, PLEs are not just collection of artifacts; they can also integrate individuals, groups and communities that impact their nature, features, and evolving structure. Finally, one should underline that PLEs are not unique, even for a given individual. People continuously move from one PLE to another when migrating to different learning contexts. The continuity of the activities, especially the learning activities, has to be ensured and sustained not only within a PLE, but also across PLEs if learners wish to do so. Conversely, learners may also want to keep a clear separation between some of their PLEs and the associated groups or communities. This vision of the exploitation of Web-based mashed-up PLEs in a personal learning framework constitutes 
the underlying hypotheses for requirements elicitation as presented in the next section.

In the ROLE European project mentioned in Section I, a multidimensional approach has been implemented to elicit the functional and non-functional requirements of personal learning environments. A range of conventional as well as emergent methods and tools for capturing and analyzing requirements have been deployed, including Web-based focus groups (testbed-oriented, longitudinal studies), workshops, questionnaires, interviews, and online communities of practice (CoP). Specifically, focus groups and $\mathrm{CoP}$ are consistent with the principles of participatory design, which is underpinned by the philosophy of user-centered design. Furthermore, CoP aligns with the emerging social requirements engineering approach [10][11]. Whilst the requirements engineering process in ROLE is ongoing, some interesting findings could already be derived from two workshops conducted and will be elaborated subsequently.

\section{REQUIREMENTS ANALYSIS: PRELIMINARY FINDINGS}

Instead of carrying out long ethnographical field studies with learners, a hybrid strategy employing both top-down (e.g., expert reviews) and bottom-up approaches (i.e., end-user focus groups) has been adopted. The intention is to actively involve all stakeholders during the entire design and development process. Scientific discourses among experienced researchers and practitioners in the field of technology enhanced learning (TEL) have been conducted through workshops and interviews. One of the workshops was held in May 2009 in which 26 ROLE project partners were involved to share their ideas and visions about responsive open learning environments. The academic backgrounds of the workshop participants were heterogeneous, including engineering, computer science, mathematics, education, psychology, management, etc. Most of them were experienced in interdisciplinary collaborations in the context of TEL. In the workshop, a series of short presentations outlining the main concepts of respective visions were followed by small group discussions where participants were asked to complete a template to describe pedagogical and technical approaches that were deemed essential for responsive open learning environments and to derive requirements from the descriptions.

The workshop materials were analyzed. Interestingly enough, during the presentations and discussions, the term personal learning environment was employed so frequently that it almost became synonymous with responsive open learning environment. However, strictly speaking, there are nuances between these two closely related notions. Here we do not delve into deeper discussion on this issue, but draw on the outcomes of the workshop that has incidentally set its focus on PLEs. The range of approaches and issues addressed during the workshop was diverse. Most commonly mentioned pedagogical and technical concepts for personal learning environments are depicted in Figure 1 and Figure 2 in the form of tag clouds, respectively. Considering the scope of the paper, we discuss only those that are highlighted in both figures.

The most important pedagogical aspect pointed out by the workshop participants is the integration of PLEs in a self- directed or self-regulated (the subtle differences between these two terms are out of the scope of this paper) learning framework where the communities play a critical role, as illustrated by the terms 'community-based', 'collaborative' and 'social'. The term 'activity-based learning' covers both individual and collaborative aspects of learning activities. Closely associated with the key concern of self-regulated learning is motivation. The term 'adaptive' has instigated interesting discussions about user-driven vs. system-driven recommendation.

The most important technical aspect pointed out by the experts is the usability of PLE widgets in a free integration framework. Not surprisingly, 'Web 2.0' and the associated concept of 'collective intelligence' are among the frequently mentioned technical terms. Open standards such as OpenSocial and XMPP (Extensible Messaging and Presence Protocol) were addressed alongside with the discussion about semantic interoperability. The term "cockpit" is used metaphorically to refer to the typical graphical user interface template of mash up platforms.

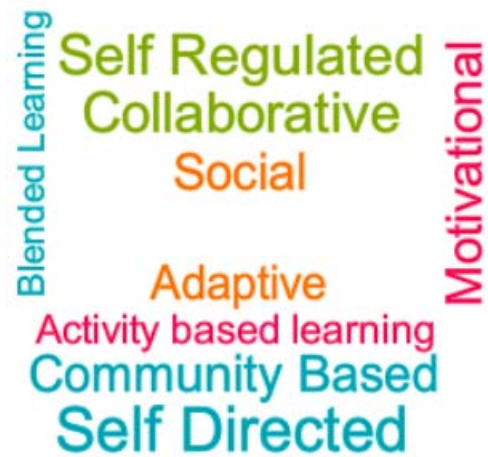

Figure 1. Tag cloud of pedagogical approaches relevant to PLE deployment.

\section{Open Standards}

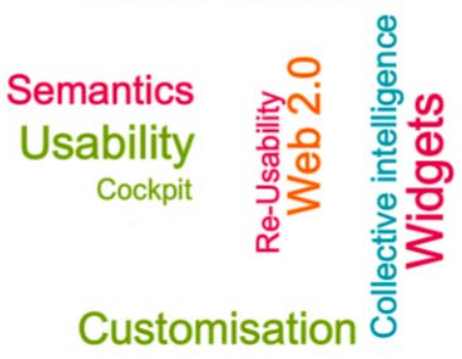

Figure 2. Tag cloud of technical approaches relevant to PLE deployment.

Some interesting discussions also occurred regarding the necessity and the way to integrate learning paths or sequences of activities (as considered in pedagogical design) in a selfdirected learning and PLE framework. No consensus emerged on this issue. However, this discussion led to a clear implication that PLEs are not persistent environments. They should evolve according to the learner's objectives and achievements, as well as competence management requirements. 
In a follow-up workshop held in July 2009, twelve PLE and technology enhanced learning experts from all around the world external to the ROLE project helped to better specify key PLE features by contributing to the requirements elicitation and refinement process. They, together with 21 ROLE project partners, discussed in detail the two key concepts: responsiveness and openness. Specifically, the notion of PLE responsiveness was defined as "the ability of the learner to successfully configure the learning environment based on recommendations, adaptations and personalization". As adaptive learning systems strongly rely on centralized user models and learning styles, it appears that system-driven adaptive features should not be a priority in PLEs. It could even be counter productive to their success by giving the impression to the learners that they lose control. As a conclusion, a stronger focus on user-driven recommendation and personalization (preferences) should be considered.

Results from analyzing the data collected in the aforementioned two workshops corroborate the assumption about the need to enrich or even replace monolithic learning environments with a highly flexible, responsive and customizable environment according to the needs of the learner. The data analysis has also led to the initial set of pedagogical and technological requirements for the ROLE project. Pedagogically, it is important to incorporate design decisions around the concepts of self-regulated learning [12] with the intention to foster communities of practices. However, the range of learning tools, content, communities and services on the Web are so huge that it could become an overwhelmingly challenging task for a learner to decide what to use when, how and why. Hence, ROLE aims to provide learners with support for building a personal learning environment and learning with it in a pedagogically meaningful way. One way currently considered by ROLE is to provide users with pre-built PLE templates accompanied with screencasts showing how and why the templates can be assembled. Traditionally, learners within formal educational environment are used to instructor-led, organisational monolithic learning environments. Over the years, this didactic approach has given way to a more constructive self-regulated learning approach to facilitate life long learning. Technologically, an architecture and interoperability framework enabling the composition and federation of different learning services is required. An approach to integrating services, tools, and data relevant for a learner is deemed necessary as well.

Of particular importance is the notion of usability, which was regarded by most of the workshop participants as a very significant quality attribute for the integrated learning services and other outcomes of ROLE. Specifically, a comment from one of the PLE experts highlights the concern. " .... even if you can solve the deliverability problems it would be great but some people can't deal with a PLE, how do they construct it....". However, a usability study performed by Silva and Dix [13] indicates that the popular social networking website YouTube has some major usability issues despite its popularity. Paradoxically, user satisfaction is nevertheless acceptably high; it thus raises a question on the necessity of rigorous usability. This claim is confronted with an counterargument put forward by Rigutti and Paoletti [14] who suggest that users might overlook the usability factor when a technology is new, satisfies their needs and there are no competing products. However, as the technology matures and more products are developed, products that are highly usable will sustain. Through ROLE we are attempting to deliver a novel approach wherein learners will be able to assemble their own PLEs. As this is a new approach to learning in a more de-centralized form, the uptake of such an approach at an early stage is critical.

Through the different requirements capturing activities mentioned above, a set of testbed-specific and some general use cases have been documented. Consequently, about 50 functional and 15 non-functional requirements (e.g. usability, privacy, trust, security) have been identified. The functional requirements are sub-divided into three groups in terms of service types: learning domain/planning (e.g. assessment, tracking), core (e.g. authentication, authorization), and communication/collaboration (e.g. chat, calendaring). It is imperative to prioritize these requirements, thereby enabling the development teams to manage their tasks efficiently and effectively. We have adapted the Kano analysis approach [15]. Accordingly, requirements should be prioritized with regard to user satisfaction - a quality dimension that is upheld in the ROLE project. With the simplified analysis scheme, we have categorized each requirement with one of two values - "must have" and "recommended". As requirements capture, analysis and validation will be a continuous process throughout the project's lifetime, the preliminary findings reported above will be subject to further refinement.

\section{PARTICIPATORY DESIGN}

Concurrently with the conversations between experts, five testbeds of complementary nature have been selected to elicit requirements at transitions between various learning settings. In these testbeds, a participatory design approach [16] is implemented under the collaboration of testbed coordinators, learner representatives, educational experts, and potential services providers to offer prototypes of PLE service bundles that could fulfill learners' expectations and strengthen their current practices. It has been demonstrated in previous similar initiatives that a proper selection of the stakeholders for the participatory design implementation is essential [17]. Especially, user and service mediators with similar decision power have to be selected to enable balanced negotiation of meaning [18] and construction of usefulness [19] regarding the requested services by the users or proposed services by the development teams. This process is also instrumental for the appropriation and the organic spreading of the solutions on the Webosphere.

Three testbeds related to higher education are discussed below. The two other testbeds are related to professional learning and are consequently outside the scope of this paper. One should however mention that a clear requirement of one of the two omitted testbeds is the necessity to make the LMS interoperable with the upcoming PLEs to enable a smooth transition between proprietary and open environments or contexts. 
The first testbed deals with the use of a Web 2.0 Knowledge Map in a course on programming techniques at the RWTH Aachen University. The main objective is to couple the Knowledge Map service with a message board within a PLE in order to enable contextual discussions and map auditing between students and alumni working in software companies. This approach should bring added value to the course in terms of motivation, content and interaction. It should also ease the transition of students between the academic and the professional world.

The second testbed deals with second-language learning in a multicultural framework and in a continuing education context at the Shanghai Jiao Tong University (SJTU). The main objective is to enable live discussions with native speakers and personal practice at transition between jobs, thanks to the integration in the PLE of tools that can exchange data, such as online dictionaries, pronunciation, microblogging, videoconferencing, and multimedia discussion tools. In a political context where social sites are often blocked, a PLE in which services can easily be replaced by equivalent non-blocked ones is essential. The integration with mobile phones is also important as part of the activities is carried out at distance. The current PLE instantiation shown in Figure 3 is implemented using Liferay Portal Community Edition, (www.liferay.com).

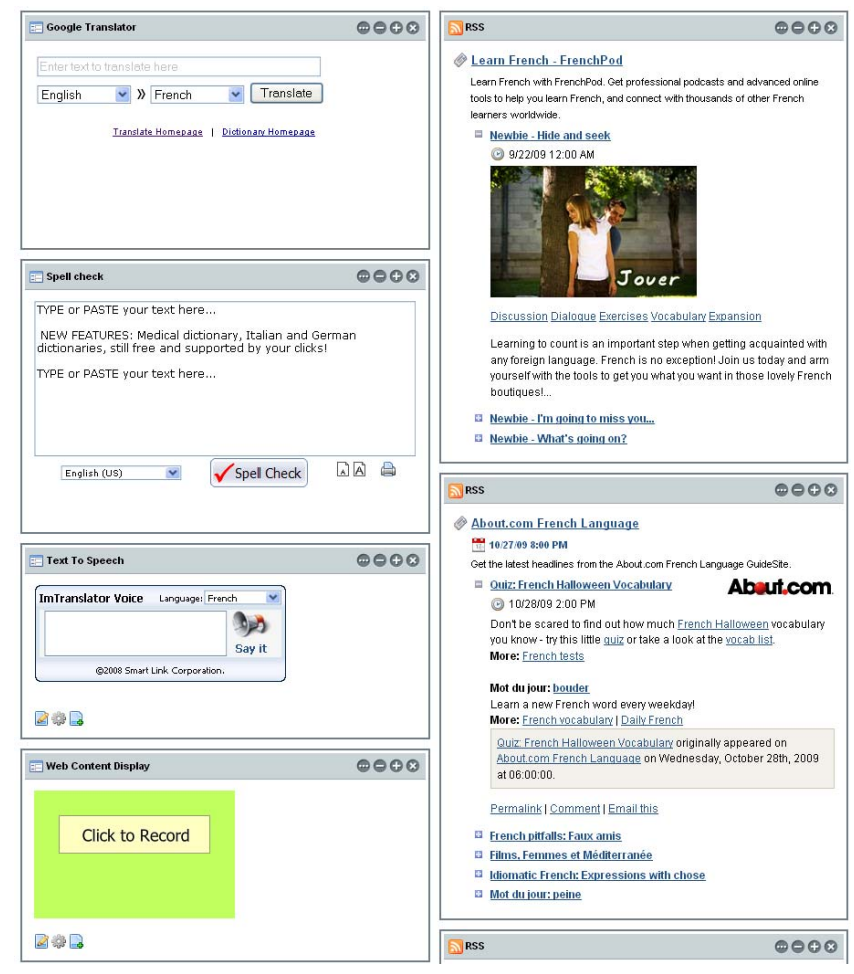

Figure 3. Screenshot of the PLE used in a French as a second language class at the Shanghai Jiao Tong University (courtesy of C. Ullrich, SJTU).

The third testbed deals with the exploitation of the OpenLearn learning space offered by the UK Open University for users in transition between informal and formal learning. Its objective is to progressively switch from a course-centric to a student-centric structure to better fulfill the users' repurposing needs, and to boost open content delivery technologies. It also aims at enabling any online learner to get support from a community of learners with similar backgrounds and goals. As it already targets educators and institutions, it is an interesting testbed to investigate the similarities between the educators' and the learners' integration approaches to combining resources in PLEs for sharing purposes or for personal usage.

Due to the heterogeneity of the testbed end users and the fact that they are geographically widely distributed; it is deemed cost-effective to facilitate the focus group discussions using a Web-based environment. The open source elgg (http://elgg.org) based social networking environment was selected as it offers all the basic Web 2.0 tools (blogs, wikis, discussion forums, social bookmarking, micro-blogging, etc) in a widget-based environment (Figure 4).

This widget-based environment enables the users to visualize what ROLE aims to achieve beyond the feature sets of similar systems currently available. It is expected that with each personal learning services bundle release, the focus groups will provide feedback on various aspects of the respective prototypes, thereby enabling us to collect evaluation data and identify potential issues that may result in refined requirements. Currently, the active focus groups are all enduser-oriented. These end-users (students, life-long learners) participating in the focus groups actively use the current learning environment provided by the test-bed institutions. The test-bed leaders have recruited the participants with the aim to provide a fairly representative sample. However, we plan to convene an expert-oriented focus group at a later stage when required. The rationale for putting together an expert-oriented focus group (internal as well as external) is to negotiate unresolved issues identified through other methods and to validate outcomes and findings from a top-down point of view.

Interestingly, the most requested features in the three described testbeds are a collaborative recommendation system proposing knowledgeable and trustful peers to interact with, and an integrated service for live interaction with them (such as Skype or FlashMeeting: http://flashmeeting.open.ac.uk). Knowledge mapping solution is also mentioned twice, maybe as an emerging Swiss knife of the knowledge worker dealing with information, ideas and competences (gap) management challenges. This low number of requested tools confirms previous finding [20] showing that typically a couple of services are used simultaneously and should be able to communicate synchronously for actual learning activities. This is coherent with the physical dimensional constraints of computer displays or Web browser windows when switching too often between contexts has to be avoided for minimizing cognitive overload. Communication with other services used at other stages in the learning process can rely on asynchronous data exchange. The XMPP protocol (http://xmpp.org) seems a promising solution for enabling communication and interoperability in the PLE framework. Various recommendation models and techniques are currently investigated in the ROLE project, including contextual collaborative solutions relying on users' weighted trust network. They will be reported in further publications.

To summarize, designing PLEs nowadays does not consist in developing one additional learning management system or 
Web 2.0 social software applications, but it rather concentrates on providing the underlying infrastructure and recommendation solutions that rely on proper trust and reputation models mapped to the continuously evolving social and institutional contexts. Such solutions should enable users to aggregate in their own environments empowering technologies, communities, learning resources, activities and Web tools. The contextualization and repurposing of the aggregated environments for learning should be enabled according to implicit or explicit learning strategies or goals. The recommendation should be self-directed by unobtrusively involving the learner in the process, and by taking into account his/her learning contexts and interests, as well as his/her network of trusties.

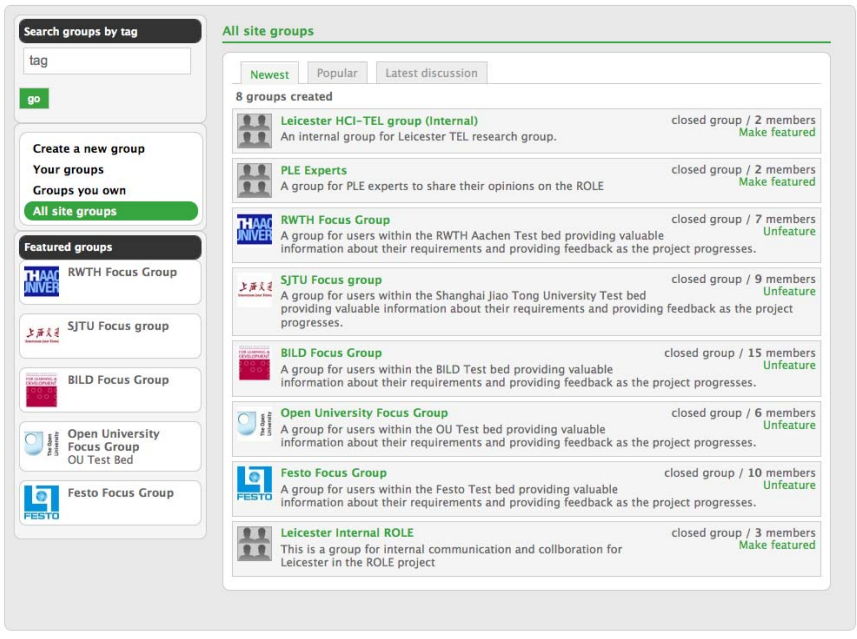

Figure 4. Web 2.0 based Focus Group facilitation.

\section{GRAAASP SOCIAL SOFTWARE PROTOTYPE}

Successful Web 2.0 solutions result from a deconstructionreconstruction process of the traditional collaborative learning practices and artifacts in order to extract essential features; followed by the implementation of a proper participatory design approach.

A previous attempt to develop and exploit a social software platform called eLogbook as a knowledge management platform in communities of practice and as a collaborative learning environment for engineering education gave good results in terms of integration of services (such as simulation and remote experimentation applets), but did not spread as expected outside the Swiss Federal Institute of Technology in Lausanne (EPFL) where it was developed due to usability reasons [21]. Specifically, it did not meet the requirements elicited in the previous section in terms of recommendation and relation with people. Only internal resources and registered members were accessible through a search feature. Invitation of external people was possible but required too much configuration and acceptation steps to be completed quickly. Also, most users did not understand its innovative graphical user interface (GUI) metaphor. As a matter of fact, this partial failure shows a pitfall associated with the participatory design approach that was implemented to design eLogbook in the framework of the Palette European Project (http://palette.ercim.org). The negotiation between the user and the developer mediators ended up with too many features that were integrated detrimentally to the GUI tuning. This problem can be avoided by pushing further the Web 2.0 philosophy of developing only wrappers to integrate as much as possible existing services or widgets in PLEs.

The Graaasp social software introduced in this section is an evolution of eLogbook hopefully correcting the flaws of the latter and that can be described as a Web 2.0 contextual aggregator. Graaasp is built on the 3A interaction model [22] that is particularly focused on describing and designing social and collaborative environments. The presence of three "a"s in Graaasp name is a reminder of this underlying model.

The 3A model accounts for three main constructs or entities: Actors are entities capable of initiating an event in a collaborative environment. They can be humans as well as virtual agents. Actors create collaboration spaces where they conduct Group Activities to reach specific objectives. In each of these activities, actors can take different roles, each of which consisting of a label and an associated set of rights. Furthermore, actors produce, edit, share and annotate Assets in order to meet activities objectives. Assets can consist of simple text files, RSS feeds, wikis, videos or audio files. The model accounts for Web 2.0 features: entities can be tagged, shared, commented, linked together and rated. As an aggregator of $3 \mathrm{~A}$ entities, Graaasp can serve not only as a networking platform, a repository of assets and an activity management system, but also as a mounting space bringing together content and services from other Web 2.0 applications and social platforms.

While keeping the 3A model internally, Graaasp displays separately in its GUI physical and virtual actors, which are labeled as People and Tools, respectively. The main characteristic of the Graaasp GUI is its ability to enforce contextual aggregation of its four categories of entity, i.e. People, Spaces (group activities), Assets and Tools (widgets, applets). Any entity dragged and dropped in the main area (1) becomes the current context of aggregation and interaction (Figure 5). The content of the associated columns (2, 3, 4 and (5) is automatically updated to only display entities with direct links to the chosen context. Direct links are relations explicitly made by users. New links with the current context can be created simply by dragging and dropping new entities on top of its dedicated grey banner area (Drop here to link). The new entities can be preselected favorites ( $(\boldsymbol{\theta})$ or entities kept in the clipboard (8). They can also result from a search or be recommended by the systems according to user preferences ( 6 or $\boldsymbol{9})$. The recommendation takes into account all the direct and indirect links existing between Graaasp entities the user has access to and the current context. Indirect links can be common tags or links going through other entities (like two actors using the same tool or having collected the same asset in a space they belong to). 


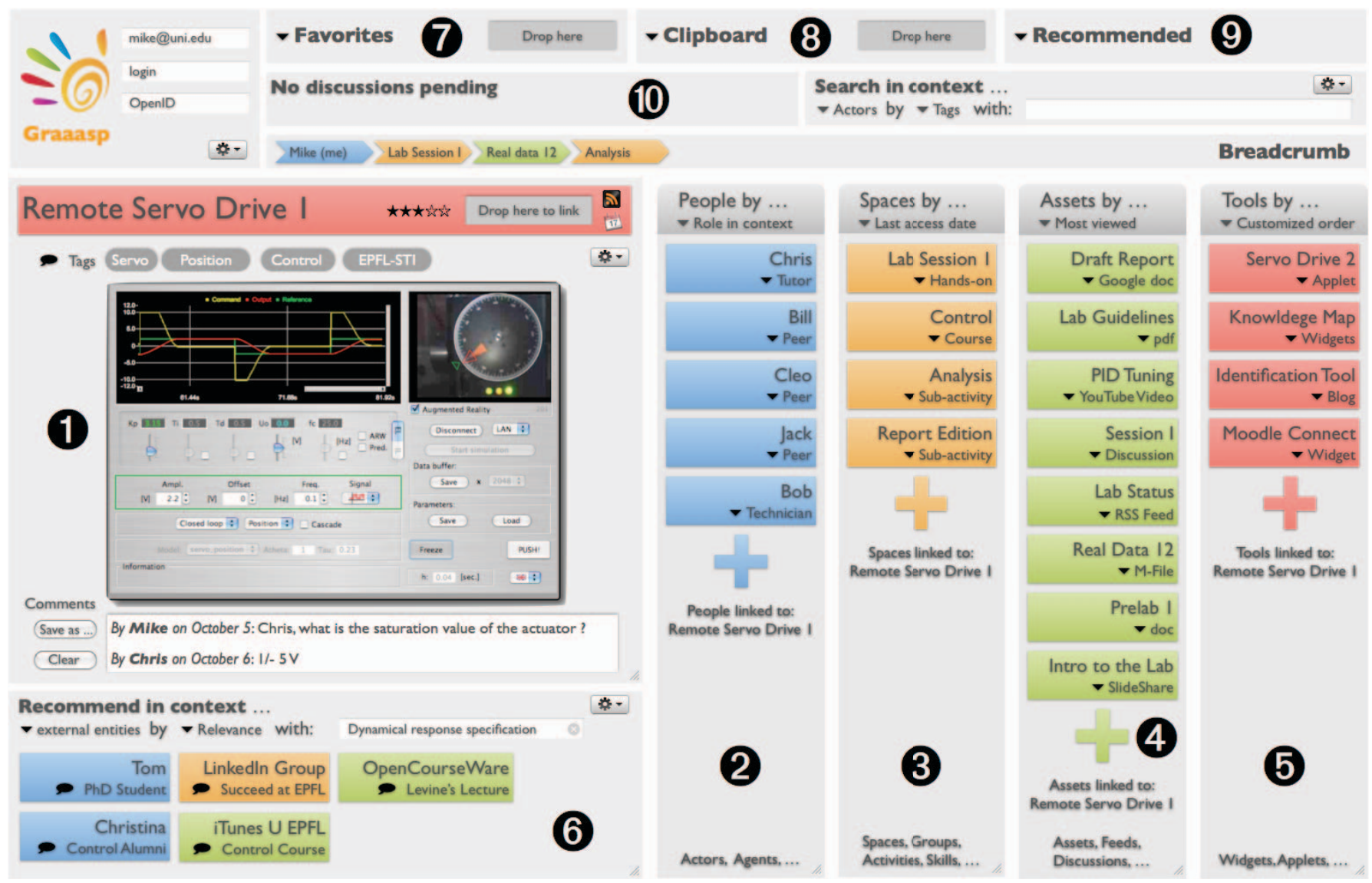

Figure 5. Recent Mock-up of the Graaasp social software (the current prototype is accessible at http://graaasp.epfl.ch).

The breadcrumb area displays the recent entities successively selected as context. The content of the columns can be displayed in predefined order (creation or last access dates, names, roles, so on and so forth) or in a customized order that can reflect the relative importance of the entities in the given context. Each category of entity supports different types that have different properties. For example, an asset can display the postings corresponding to a RSS feed, while another asset can just contain a static document.

Grabbing the nature and the implication of the various existing types of links may be challenging for users. However, the graphical representation should help in visualizing the existing relationships and in exploiting the contextual navigation. Hence, Graaasp uses pre-defined settings to translate drag-and-drop actions into meaningful relations. As an example, if a blue rectangle representing a person is dropped onto a space selected as current context, this person is invited to join this space as member with the relevant rights (this member role can later be modified). If a green rectangle representing an asset is dropped onto a person selected as current context, this asset is shared between the user and that person. The implications of the different drag-and-drop actions are currently adjusted and validated with volunteers.

The Graaasp internal recommendation engine will show its full potential in 2010 when resources for other social platforms will be mounted as external $3 \mathrm{~A}$ entities. Currently, specific external resources can already be imported into Graaasp. As example, a YouTube video or a SlideShare presentation can be imported into Graaasp as an asset just by clicking on the Graaasp it! bookmarklet. A bookmarklet is an applet stored as the URL of a bookmark in a Web browser that can be executed at anytime when visiting supported websites to import interesting resources in Graaasp. Plug-ins to support additional websites can easily be implemented on the Graaasp server side, broadening in such a way the scope of the bookmarklet without the need to reinstall it every time improvements are made.

It is not clear yet whether users will indeed adopt Graaasp as a PLE. Further validation with educators and learners should be conducted. However, its design clearly supports the contextual aggregation of resources, including widgets in the tool category, and communities for general knowledge management or learning purposes. In that sense, it can be seen as a PLE generator that could be used by educators to prepare and propose potentially useful learning service bundles to their students. These bundles can be exploited directly in Graaasp or possibly exported in the future to other platforms. In such a way, the experience and the educators' accredited resources and networks can be embedded in shared PLE configurations that can include a predefined set of tools to be possibly exploited by the students. Hence, the burden for the students to build from scratch their PLEs may be reduced. These 
configurations can also integrate recommended experts using OpenSocial [23] technology.

\section{EXPLOITATION SCENARIO IN ENGINEERING EDUCATION}

As a tentative exploitation scenario of the Graaasp social software as PLE in engineering education, one can consider the example of a student carrying out collaborative laboratory activities in control (a mandatory course offered at EPFL to students in mechanical, electrical, and micro engineering).

The students have three hands-on laboratory modules to complete during the last semester of their bachelor program. They can access the laboratory experiments directly on campus once a week, or remotely, 24 hours a day, 7 days a week. The typical laboratory setup introduced to practice position and speed control is a servo drive. An applet is available for local or remote data acquisition and control of this system [24].

Mike, a student in electrical engineering was already using Graaasp to manage the EPFL Jazz band. He decided to use the same social software to manage the control laboratory modules with peers. As EPFL strongly support the development of autonomy and teamwork skills, he knew that he could freely choose the other students he wished to work with. After a discussion at the cafeteria, Cleo, Bill and him decided to carry out the lab together. They had complementary competences and had already collaborated effectively in other contexts.

Mike connected first to Graaasp to create a new space called Lab Session 1 in order to support the lab activities associated with the first module to complete. He then invited Cleo and Bill to join this activity as peers. He also searched for available servo drives and linked the applet corresponding to the Remote Servo Drive 1 as tool in his Lab Session 1 space. He later picked Chris as tutor as he had got good feedback on his competences from Jack, a member of the Jazz band and master student in mechanical engineering who took the same lab session the previous year. Mike decided to invite Jack in the space, just in case further interaction with him would be required. Chris accepted the invitation to coach Mike's team, as he had not yet reached his quota of students. As a tutor, Chris is not in charge of the team evaluation in order not to refrain them from discussing the subject matter openly. Mike also added tools and assets useful for the lab in the space, including his favorite Knowledge Map widget, a nice YouTube video showing how to tune the PID controller they have to work with, and the RSS feed which is updated with the current status of the actual lab experiments, just to be notified in case their selected servo drive will be unplugged for maintenance. He also created a Google doc for the collaborative editing of the report and shared it within the main space (Lab Session 1) and its corresponding sub-activity space.

When Mike started his first real experiment by dropping the Remote Servo Drive 1 applet as context, Bob, the technician in charge of the maintenance appears automatically as linked person. Also, three additional tools linked directly and publically to the applet by Bob became visible. An additional servo drive that can be used as backup, an Identification Tool for the processing of the measurements acquired on the servo drive and the Moodle Connect tool enabling the storage of the data for the users that are still using the old LMS of the university.
Mike got a list of additional relevant entities related to his context and with the additional keywords he gave, including the podcasts of the EPFL control course on iTunes $U$ and the slides of a related MIT course available on OpenCourseWare, which gave him an additional perspective on the subject matter.

As Cleo was so happy to get all the material necessary to complete the lab assignments at a single but open place, and to be able to keep it even after the end of the course, she decided to continue to use and populate Graaasp for most of her other social and academic activities. Lately, she exported one of her space dedicated to Philosophy just by one click to iGoogle, a platform some of her friends preferred to use.

\section{CONCLUDING REMARKS}

This paper discussed the motivations and the challenges associated with the introduction of personal learning environments in higher engineering education.

In addition to highlighting the need for a fundamental change necessary in the educational paradigm to better account and support personal learning, essential pedagogical and technological requirements pertaining to PLEs are pointed out. Some of the issues identified and highlighted in Figure 1 and 2 are still being discussed within ROLE's TEL community. These issues (mostly pertaining to self-regulated learning, community based learning, recommendation, inter-operability standards, PLE usability) are contentious in nature and will require diligent negotiation among stakeholders to reach an acceptable resolution. Especially, usability is elicited as one of the most challenging features of personal learning environments, together with the need of powerful recommendation capability to help learners find relevant resources and people in the Web 2.0 realm. Hence, the reader must bear in mind that ROLE is an ongoing project and this paper reports some of the issues that we have identified so far and presents a preliminary PLE prototype with potential resolutions for some of the open issues like recommendation or community cohesiveness.

An example of a novel Web 2.0 social software that can be exploited in engineering education is presented, together with an implementation scenario for laboratory activities associated with a control course. This example shows how both the pedagogical and the learning environment design can be adjusted for a better integration of formal and informal learning practices.

As an emerging topic in field of technology enhanced learning, the design and development of open and responsive PLEs is deemed challenging. A number of controversies entail further scientific discourses and more empirical validations. Amongst others, we name several examples: striving the balance between system-driven and user-driven personalization mechanisms (cf. the privacy issue pertinent to user profiling), deepening the understanding of trust-building enablers and integrating them into recommendation protocols, and identifying viable means to sustain the development of everaugmenting widget-landscape. We aim to tackle these challenges in our future research work. 


\section{ACKNOWLEDGMENT}

Dr. Christophe Salzmann, Dr. Stéphane Sire, Ms Sandy El Helou and Mr Evgeny Bogdanov have contributed to the recent research and prototypes presented in this paper. The research work described in this paper is partially funded through the ROLE Integrated Project; part of the Seventh Framework Programme for Research and Technological Development (FP7) of the European Union in Information and Communication Technologies.

\section{REFERENCES}

[1] G. Attwell, "The personal learning environments - The future of eLearning?" eLearning Papers, vol. 2(1), 2007.

[2] S. El Helou, D. Gillet, C. Salzmann, and S. Sire, "3A contextual ranking system: Simultaneously ranking actors, assets \& collaborative activities," in Proc. 3rd ACM Conference on Recommender Systems, New York City, NY, USA, 2009.

[3] J. E. Hirsch, "An index to quantify an individual's scientific research output," in Proc. National Academy of Sciences, USA, vol. 102(46), 2005, pp. 16569-16572.

[4] M. Weller, "The distance from isolation: Why communities are the logical conclusion in e-learning," Computers \& Education, vol. 49(2), September 2007, pp. 148-159.

[5] J. Vassileva, "Towards Social Learning Environments," IEEE Transactions on Learning Technologies, vol. 1(4), 2008, pp. 199-213

[6] R. Land, and S. Bayne, "Social technologies in higher education: Authorship, subjectivity and temporality," In Proc. of the 6th International Conference on Networked Learning, 2008, pp. 675-681.

[7] C. Philip, "Linking thinking: self-directed learning in the digital age," DEST, 2004, viii, 344 p., http://www.voced.edu.au/docs/ dest/TD_AG_80_02.pdf (viewed November 2009).

[8] M. Johnson, and O. Liber, "The Personal learning environment and the human condition: from theory to teaching practice," Interactive Learning Environments, 16(1), April 2008, pp. 3-15.

[9] S. Wilson, O. Liber, M. Johnson, P. Beauvoir, P. Sharples, C. Milligan, "Personal learning environments: Challenging the dominant design of educational systems," Journal of e-Learning and Knowledge Society, vol. 2, 2007.

[10] S. Lohmann, S. Dietzold, P. Heim, and N. Heino, "A Web platform for social requirements engineering,” In: J. Münch, P. Liggesmeyer (Ed.), "Software Engineering 2009 - Workshopband," Germany, Kaiserslautern, March 2-6, ISBN 978-3-88579-244-4, 2009.

[11] V. Bryl, P. Giorgini, and J. Mylopoulos, "Designing socio-technical systems: from stakeholder goals to social networks," Requirements Engineering, 14(1), 2009, pp. 47-70.
[12] B. J. Zimmerman, "Becoming a self-regulated learner: An Overview," Theory into Practice, vol. 41(2), 2002, 64-70.

[13] P. Silva, and A. Dix, "Usability - not as we know it!," In Proc. of the 21st BCS HCI Group Conference HCI 2007, September 3-7, 2007, Lancaster University, UK.

[14] S. Rigutti, and G. Paoletti, "Web 2.0: which usability issues?," Journal of e-Learning and Knowledge Society, vol. 4(2), 2008, pp. 229 -234.

[15] N. Kano, N. Seraku, F. Takahashi, and S. Tsuji, "Attractive quality and must-be quality," The Journal of the Japanese Society for Quality Control, vol. 14 (2), 1984, pp. 39-48.

[16] S. Bødker, and O. S. Iversen, O. S. " Staging a professional participatory design practice: moving PD beyond the initial fascination of user involvement." In Proc. of the Second Nordic Conference on HumanComputer interaction, NordiCHI '02, vol. 31, ACM, New York, NY, 2002, pp. 11-18.

[17] S. El Helou, M. Tzagarakis, D. Gillet, N. Karacapilidis, and C. Y. Man, "Participatory design for awareness features: Enhancing interaction in communities of practice," In Proc. of the 6th International Conference on Networked Learning, Halkidiki, Greece, May 5-6, 2008.

[18] L. Dirckinck-Holmfeld, "Designing for collaboration and mutual negotiation of meaning - boundary objects in networked learning environments," In Proc. of the Fifth International Conference on Networked Learning, 2006.

[19] R. A. De Paula, "The construction of usefulness: how users and context create meaning with a social networking system," Doctoral Thesis. UMI Order Number: AAI3153816., University of Colorado at Boulder, 2004.

[20] L. Esnault, N. Karacapilidis, D. Gillet, and Ch. Vanoirbeek, "Developing interoperable collaboration services to sustain activities of communities of practice," International Conference on Information Resources Management (Conf-IRM), Niagara Falls, Ontario, Canada, May 18-20, 2008.

[21] S. El Helou, C. Salzmann, D. Gillet, and C. M. Yu, "A study of the acceptability of a Web 2.0 application by higher-education students undertaking collaborative laboratory activities," In Proc. of the 2nd International Conference on Advances in Computer-Human Interactions, 2009, pp. 117-125.

[22] S. El Helou, D. Gillet, C. Salzmann, and Y. Rekik, "Software for sustaining interaction, collaboration and learning in communities of practice," In "Solutions and innovations in Web-based technologies for augmented learning: Improved platforms, tools, and applications," Advances in Web-based Learning (AWBL) Book Series, 2009, pp. 300316.

[23] A. C. Weaver, B. B. Morrison, "Social Networking," Computer, vol. 41(2), Feb. 2008, pp. 97-100.

[24] D. Gillet, S. El Helou, C. M. Yu, and C. Salzmann, "Turning Web 2.0 Social Software into Versatile Collaborative Learning Solutions," In Proc. of the First International Conference on Advances in ComputerHuman Interaction, 2008, pp.170-176. 
